\title{
Revealing the Distribution of Aggregation-Induced Emission Nanoparticles via Dual-Modality Imaging with Fluorescence and Mass Spectrometry
}

\author{
Liucheng Mao, ${ }^{1}$ Yuming Jiang, ${ }^{2}$ Hui Ouyang, ${ }^{3}$ Yulin Feng, ${ }^{3}$ Ruoxin Li, ${ }^{1}$ Xiaoyong Zhang $\left(\mathbb{D},{ }^{4}\right.$ \\ Zongxiu Nie $\left(\mathbb{b},{ }^{2}\right.$ and Yen Wei ${ }^{1}{ }^{1}$ \\ ${ }^{1}$ The Key Laboratory of Bioorganic Phosphorus Chemistry \& Chemical Biology (Ministry of Education), Department of Chemistry, \\ Tsinghua University, Beijing 100084, China \\ ${ }^{2}$ Beijing National Laboratory for Molecular Sciences, Key Laboratory of Analytical Chemistry for Living Biosystems, \\ Institute of Chemistry, Chinese Academy of Sciences, Beijing 100190, China \\ ${ }^{3}$ State Key Laboratory of Innovative Drug and Efficient Energy-Saving Pharmaceutical Equipment, Jiangxi University of Traditional \\ Chinese Medicine, Nanchang 330006, China \\ ${ }^{4}$ Department of Chemistry, Nanchang University, 999 Xuefu Avenue, Nanchang 330031, China
}

Correspondence should be addressed to Xiaoyong Zhang; zhangxiaoyong@ncu.edu.cn, Zongxiu Nie; znie@iccas.ac.cn, and Yen Wei; weiyen@mail.tsinghua.edu.cn

Received 29 April 2021; Accepted 3 June 2021; Published 19 June 2021

Copyright ( 2021 Liucheng Mao et al. Exclusive Licensee Science and Technology Review Publishing House. Distributed under a Creative Commons Attribution License (CC BY 4.0).

\begin{abstract}
Aggregation-induced emission nanoparticles (AIE NPs) are widely used in the biomedical field. However, understanding the biological process of AIE NPs via fluorescence imaging is challenging because of the strong background and poor penetration depth. Herein, we present a novel dual-modality imaging strategy that combines fluorescence imaging and label-free laser desorption/ionization mass spectrometry imaging (LDI MSI) to map and quantify the biodistribution of AIE NPs (TPAFN-F127 NPs) by monitoring the intrinsic photoluminescence and mass spectrometry signal of the AIE molecule. We discovered that TPAFN-F127 NPs were predominantly distributed in the liver and spleen, and most gradually excreted from the body after 5 days. The accumulation and retention of TPAFN-F127 NPs in tumor sites were also confirmed in a tumor-bearing mouse model. As a proof of concept, the suborgan distribution of TPAFN-F127 NPs in the spleen was visualized by LDI MSI, and the results revealed that TPAFN-F127 NPs were mainly distributed in the red pulp of the spleen with extremely high concentrations within the marginal zone. The in vivo toxicity test demonstrated that TPAFN-F127 NPs are nontoxic for a long-term exposure. This dual-modality imaging strategy provides some insights into the fine distribution of AIE NPs and might also be extended to other polymeric NPs to evaluate their distribution and drug release behaviors in vivo.
\end{abstract}

\section{Introduction}

Luminescent materials have attracted attention for their utility in chem-/biosensors [1-3], photoelectric devices [4-6], and biomedical diagnosis or therapy [7-10]. As one of the most promising luminescent materials, aggregation-induced emission luminogens (AIEgens) are nearly nonluminescent in solution while highly luminescent in the aggregate or solid state [11]. The fascinating photoluminescence (PL) behavior and intrinsic hydrophobicity of AIEgens are particularly beneficial for the fabrication of AIEgen-based nanomaterials [12]. Typically, polymer-encapsulated AIEgen-containing nanoparticles (AIE NPs) with features such as desirable size, stable brightness, high resistance to photobleaching, and excellent biocompatibility have been used as fluorescent contrast reagents for in vitro or in vivo bioimaging and disease diagnostic reagents [13-19]. Recently, through rational design of AIEgens, AIE NPs have been endowed with multifunctionality, including photodynamic or photothermal therapeutic ability, making them suitable for theranostic applications [20-28]. As the biomedical applications of AIE NPs become more widespread, increasing concerns have been raised about their behavior in biological systems [29]. However, fluorescence imaging inevitably suffers from limitations, such as 


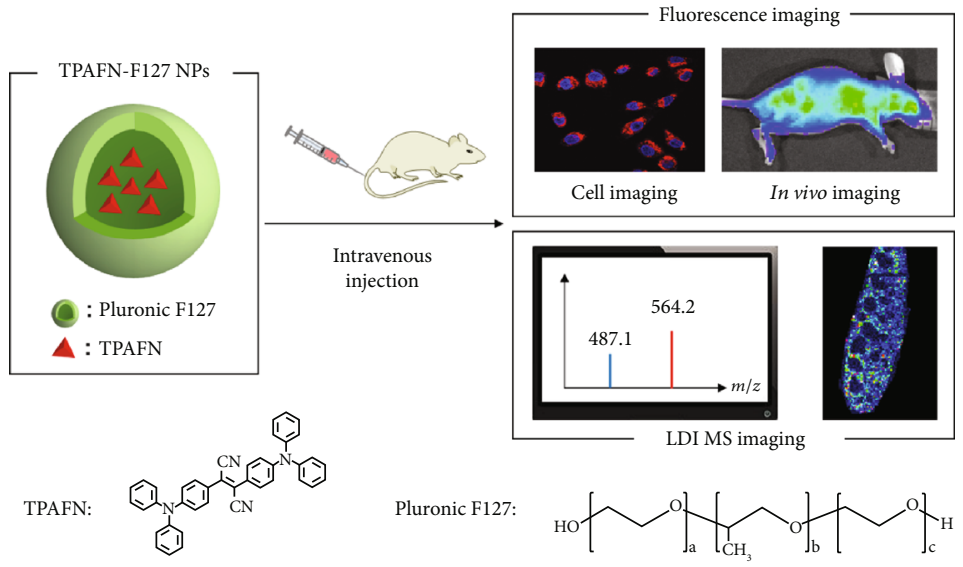

Scheme 1: Schematic of TPAFN-F127 NPs for fluorescence imaging and LDI MSI.

photobleaching, strong background, and poor penetration depth [30, 31]. Determining how to depict the fine biodistribution of AIE NPs at both the microscopic and macroscopic levels remains a big challenge. Therefore, developing a complementary analysis technology to assist fluorescence imaging for mapping and quantifying the biodistribution of AIE NPs is of great significance.

Matrix-assisted laser desorption/ionization mass spectrometry imaging (MALDI MSI) has become an accurate analysis tool for the detection and quantification of targeted compounds (e.g., nucleic acids, proteins, polysaccharides, drugs, and metabolic products) in tissue sections and for mapping their spatial distribution at the suborgan or single-cell level [32-37]. Recently, a new laser desorptio$\mathrm{n}$ /ionization mass spectrometry imaging (LDI MSI) technique that does not require a matrix has received significant attention for in vivo analysis and mapping nanomaterials (e.g., carbon nanomaterials, gold nanoparticles, and $\mathrm{MoS}_{2}$ nanosheets) based on their intrinsic fingerprint signal [38-40]. This direct and label-free method avoids the potential diffusion of target compounds and interference from biomolecules. Encouraged by these studies, we hypothesized that the proposed LDI MSI method could be employed to identify the suborgan or tissue distribution of AIE NPs by detecting the molecular weight of AIEgens, as AIE nanoparticles consist of AIEgens in the core and biocompatible matrices as shells. It is noteworthy that the combination of LDI MSI and fluorescence imaging methods can enhance both the reliability and accuracy of determination. More importantly, LDI MSI could localize the materials (even their modifications) with high resolution without any background interference. Although previous studies have reported the dual/multimodel imaging applications of AIEgen-based materials, the signals originate from different components (e.g., AIEgens and gadolinium) $[41,42]$. To the best of our knowledge, the application of AIEgens for fluorescence/mass spectrometry dual-model imaging that relies only on their intrinsic properties has not been reported thus far.

In the present work, a novel dual-modality imaging technique that combines fluorescence imaging and LDI MSI by monitoring the intrinsic photoluminescence and mass spectrometry signal of an AIEgen (named as TPAFN) is reported. Pluronic F127 was chosen as the polymer matrix to improve the water dispersibility and biocompatibility of TPAFN-F127 NPs (Scheme 1). The obtained TPAFN-F127 NPs possessed a narrow size distribution, regular spherical structure, high photostability, and good chemical stability. After evaluating their biocompatibility, fluorescence imaging was conducted. An LDI MSI approach was further employed to study the localization, distribution, and quantification of AIE nanoparticles in tissues. To the best of our knowledge, this is the first study on the biodistribution of AIE NPs by LDI MSI. We hope this research will provide a deeper insight into the biological process of AIE NPs in vivo.

\section{Results and Discussion}

2.1. Preparation and Characterization of TPAFN and TPAFN-F127 NPs. The intermediate product (FN-2Br) and target AIEgen (TPAFN) were synthesized according to the previously reported methods (Scheme S1) [43]. All synthetic products were verified by ${ }^{1} \mathrm{H} /{ }^{13} \mathrm{C} \mathrm{NMR}$ spectroscopy and mass spectrometry. The specific spectroscopic data of various products are listed in Materials and Methods, and the spectra are shown in Figures S1-S6. The optimal absorption peaks of TPAFN in toluene, tetrahydrofuran (THF), and dichloromethane (DCM) were located at 488, 480, and $492 \mathrm{~nm}$, respectively (Figure 1(a)). It is worth mentioning that the maximum emission of TPAFN shifted from $622 \mathrm{~nm}$ (toluene) to $689 \mathrm{~nm}$ (DCM) with an increase in the solvent polarity, which is indicative of the typical twisted intramolecular charge transfer (TICT) (Figure 1(b)). The fluorescence spectra of TPAFN in a THF/water solution were obtained, which is a common method for studying AIE property (Figure 1(c)). The emission of TPAFN in pure THF was relatively weak, and the PL intensity gradually declined, while the emission wavelength underwent a bathochromic shift before the water fraction $\left(f_{\mathrm{w}}\right)$ reached $60 \%$, demonstrating the TICT state [44]. However, the PL intensity was dramatically enhanced at high water ratios $\left(f_{\mathrm{w}}>60 \%\right)$ (Figure $\left.1(\mathrm{~d})\right)$. These results indicate that TPAFN possesses both TICT and AIE properties. 

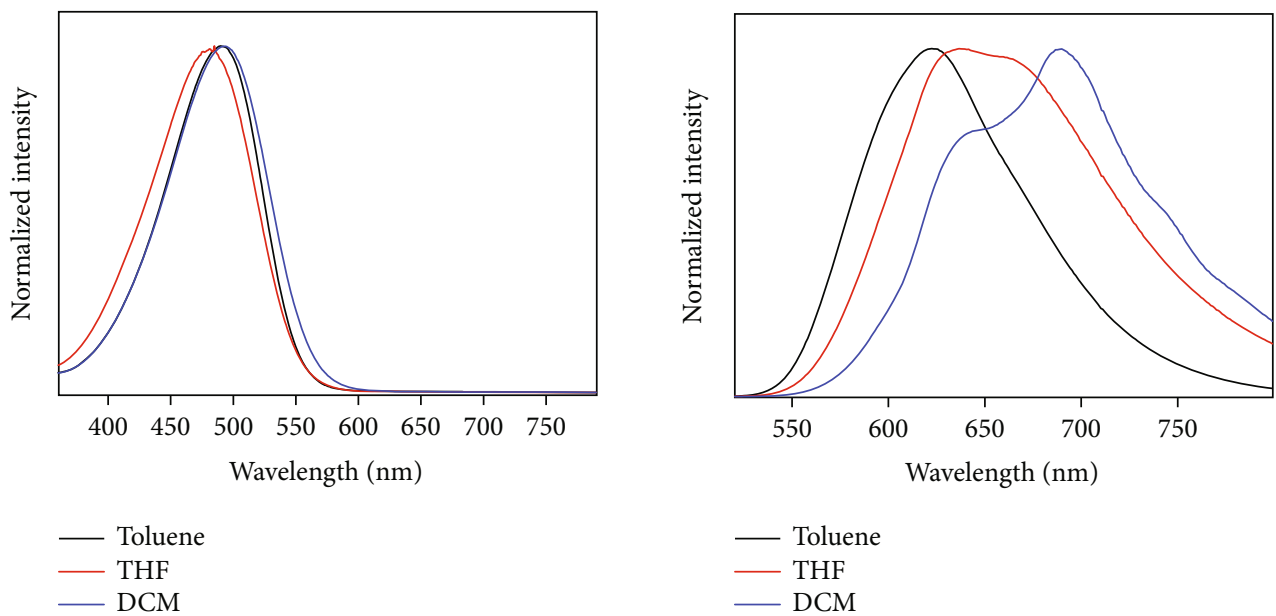

(a)

(b)
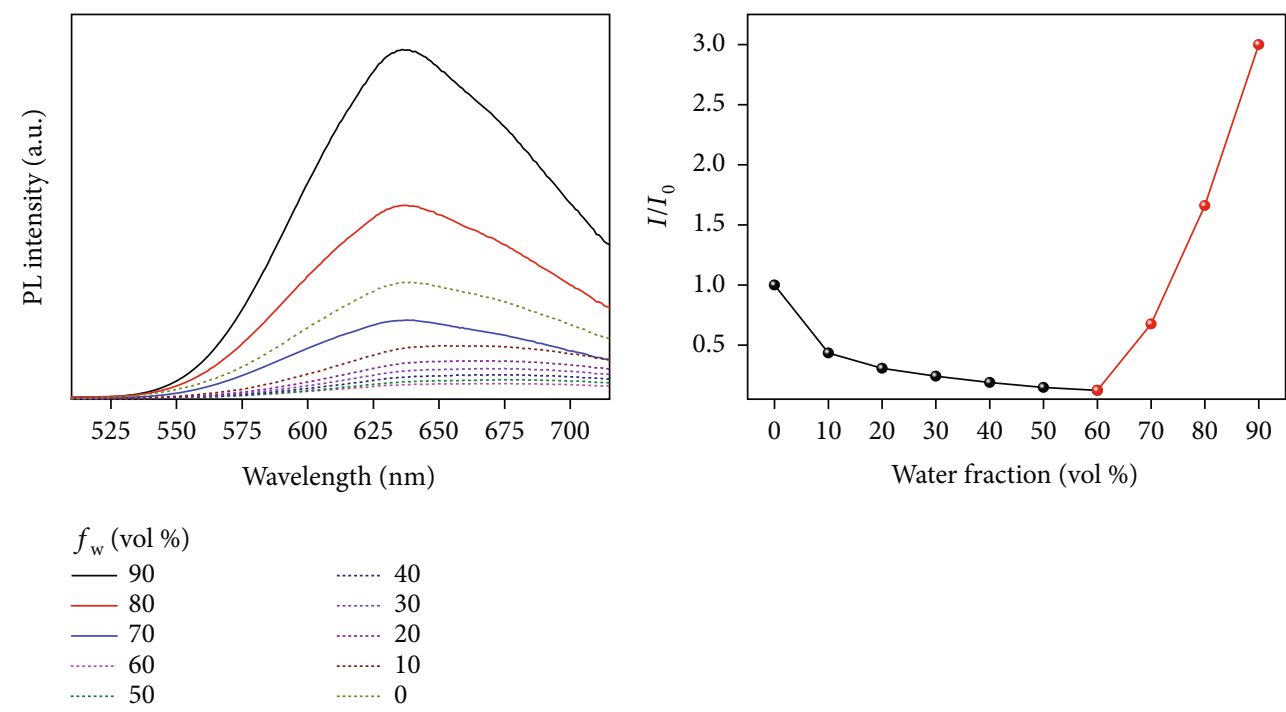

(c)

(d)

Figure 1: Photophysical properties of TPAFN. (a) Normalized UV-vis spectra of TPAFN in toluene, THF, and DCM. (b) Normalized PL spectra of TPAFN in toluene $(\mathrm{Ex}=488 \mathrm{~nm})$, THF $(480 \mathrm{~nm})$, and DCM $(492 \mathrm{~nm})$. (c) PL spectra of TPAFN in an aqueous THF solution with different water fractions $\left(f_{\mathrm{w}}\right), \lambda_{\mathrm{ex}}=480 \mathrm{~nm}$. (d) Variation in the PL intensity of TPAFN in an aqueous THF solution with different water fractions. $I_{0}$ denotes the PL intensity of TPAFN in pure THF.

The unique AIE characteristic of TPAFN is beneficial for the fabrication of AIE nanoparticles. Pluronic F127 was employed to encapsulate TPAFN via a thin-film hydration method (Scheme S2). The average size of the TPAFN-F127 NPs was $126 \mathrm{~nm}(\mathrm{PDI}=0.352$ ) (Figure 2(a)). Furthermore, TEM images indicate that the obtained TPAFN-F127 NPs possessed a spheroidal morphology and smooth surface (Figure S7). No evident changes on the hydrodynamic diameters were observed after TPAFN-F127 NPs were stored for $7 \mathrm{~d}$ (Figure $2(\mathrm{~b})$ ). The photophysical properties of TPAFN-F127 NPs were also investigated. The optimal absorption and maximum emission peaks appeared at $493 \mathrm{~nm}$ and $638 \mathrm{~nm}$, respectively (Figure 2(c)). More importantly, good photostability of TPAFN-F127 NPs was demonstrated in water (Figure 2(d)). The fluorescence intensity was barely affected when TPAFN-F127 NPs were mixed with various $\mathrm{pH}$ buffers ranging from 1 to 13
(Figure S8). These conclusions suggest that TPAFN-F127 NPs have excellent potential for biological imaging.

2.2. Cytotoxicity and Biocompatibility of TPAFN-F127 NPs. The cytotoxicity of TPAFN-F127 NPs was evaluated using L929 cells and HeLa cells through the CCK-8 assay. Negligible cytotoxicity was observed even at TPAFN-F127 NP concentrations up to $20 \mu \mathrm{g} \mathrm{mL}^{-1}$, suggesting that the NPs were biocompatible with L929 cells and HeLa cells (Figure 3(a)). Moreover, the long-term potential toxicity of TPAFN-F127 NPs was evaluated in Kunming mice. Compared to the control groups, no significant body weight variation was found in the TPAFN-F127 NPs $(250 \mu \mathrm{L}, 20 \mathrm{mg} / \mathrm{kg})$ (Figure 3(b)). Meanwhile, hematoxylin and eosin- ( $\mathrm{H} \mho \mathrm{E}-$ ) stained images of the main organs (heart, liver, spleen, lung, and kidney) revealed no evidence of inflammatory lesions in both control groups and experimental groups at day 30 of posttreatment 


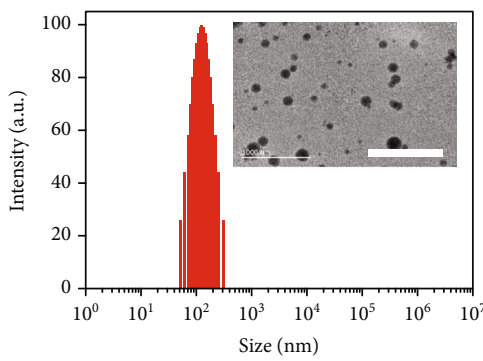

(a)

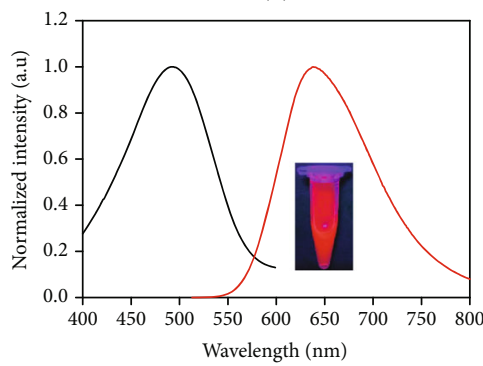

- $\mathrm{Ab}$

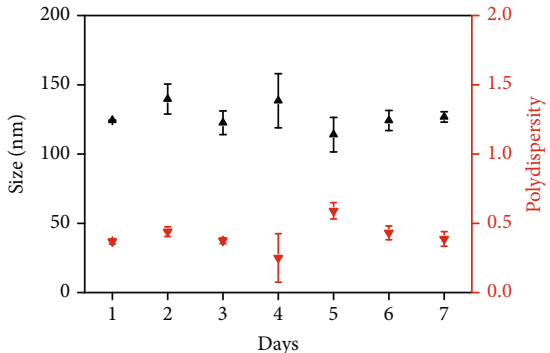

(b)

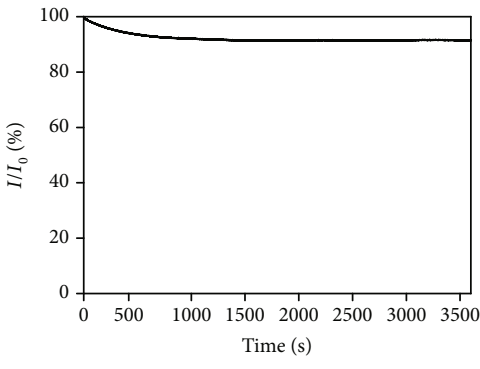

— TPAFN-F127 NPs

(d)

Figure 2: (a) Size distribution of TPAFN-F127 NPs in aqueous solution. Inset: TEM image of TPAFN-F127 NPs; scale bar: $1 \mu \mathrm{m}$. (b) Size stability of TPAFN-F127 NPs after a 7-day storage. (c) UV-vis absorption (Ab) and emission (Em) spectra of TPAFN-F127 NPs in water. Inset: PL image of TPAFN-F127 NPs acquired under $365 \mathrm{~nm}$ UV illumination at room temperature. (d) Photostability evaluation of TPAFN-F127 NPs in water under irradiation $(493 \mathrm{~nm})$ for $1 \mathrm{~h}$.

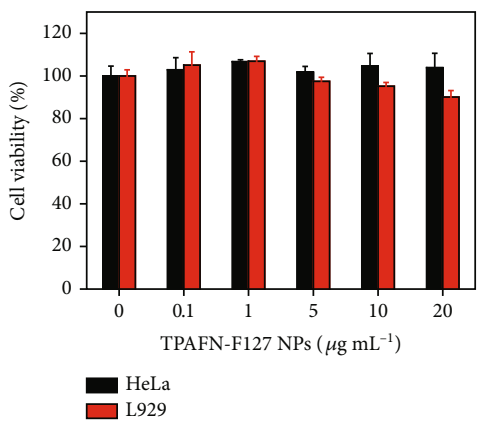

(a)

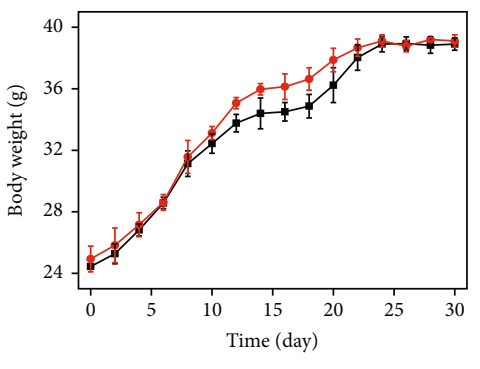

$\rightarrow$ PBS

$\rightarrow$ TPAFN-F127 NPs
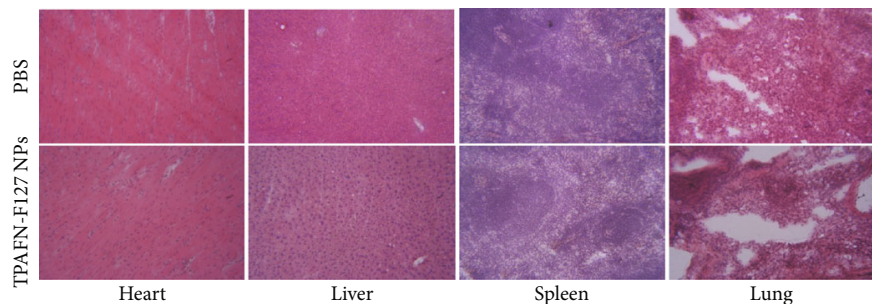

(b)

(c)

Figure 3: (a) Cell viability of L929 cells and HeLa cells incubated with TPAFN-F127 NPs at different concentrations for $24 \mathrm{~h}$. (b) Body weight variation of mice $(n=5)$ treated with $250 \mu \mathrm{L}$ PBS and TPAFN-F127 NPs $(250 \mu \mathrm{L}, 20 \mathrm{mg} / \mathrm{kg})$. (c) Representative H\&E-stained images of main organ slices collected from various groups after a 30-day treatment. Scale bar: $100 \mu \mathrm{m}$.

(Figure 3(c)). We further performed blood biochemical analysis to assess the hematological biocompatibility of TPAFNF127 NPs in mice after 7 days of treatment. As shown in Table S1, a slight statistical difference was observed in all hematological parameters, even though the dose of TPAFNF127 NPs up to $20 \mathrm{mg} / \mathrm{kg}$ demonstrated no significant inflammation. In general, the above results substantiate the biocompatibility of TPAFN-F127 NPs at an appropriate dose for biomedical applications.

2.3. Fluorescence Imaging of TPAFN-F127 NPs. The in vitro cellular uptake of TPAFN-F127 NPs was studied using 


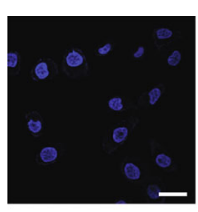

(a)

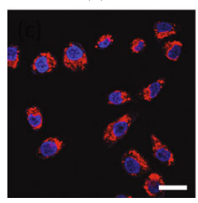

(c)

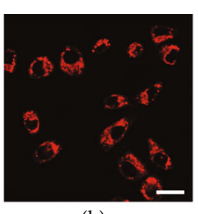

(b)

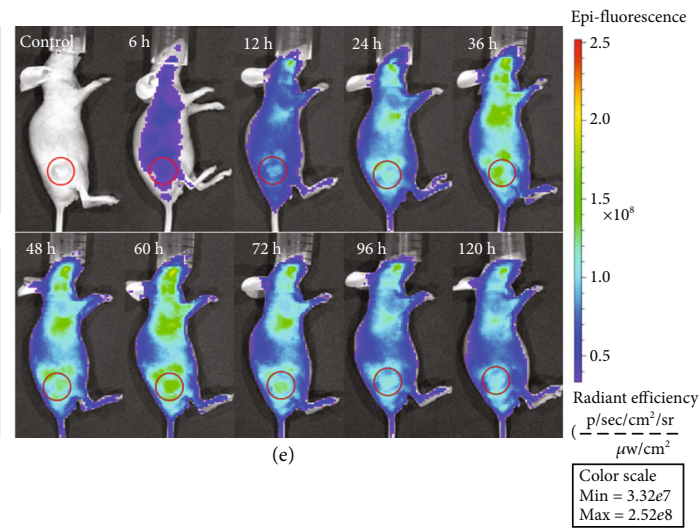

FIGURE 4: CLSM imaging of (a-d) HeLa cells incubated with TPAFN-F127 NPs for $3 \mathrm{~h}$. The concentration of TPAFN-F127 NPs is $5 \mu \mathrm{g} \mathrm{mL}^{-1}$. Scale bar: $20 \mu \mathrm{m}$. (e) In vivo fluorescence imaging of HeLa tumor-bearing mice after intravenous injection with TPAFN-F127 NPs $(200 \mu \mathrm{L}$, $10 \mathrm{mg} / \mathrm{kg}$ ). The tumor region is circled in red.

confocal laser scanning microscopy (CLSM). After HeLa and L929 cells were similarly treated with TPAFN-F127 NPs (5 $\mu \mathrm{g} \mathrm{mL}^{-1}$ ) for $3 \mathrm{~h}$, the cells were stained with Hoechst 33258. As indicated by the CLSM images in Figures 4(a)-4(d) and Figure S9, the homogeneous red fluorescence was located in the cytoplasm and the blue region in the nucleus, suggesting that TPAFN-F127 NPs were efficiently accumulated and distributed in the cytoplasm of both HeLa cells and L929 cells. These results demonstrate that TPAFN-F127 NPs are a good candidate for biological imaging.

Noninvasive in vivo fluorescence imaging was also conducted using HeLa tumor-bearing mice to assess the biodistribution and tumor accumulation of TPAFN-F127 NPs. In vivo fluorescence imaging and tumor accumulation of TPAFN-F127 NPs in HeLa tumor-bearing mice over time are shown in Figure 4(e). The PL intensity in the tumor region becomes brighter before $60 \mathrm{~h}$ postinjection, indicating that TPAFN-F127 NPs accumulated in the tumor through the enhanced permeability and retention (EPR) effect (Figure S10) $[45,46]$. It is important to note that the PL signals in the tumor region remained detectable even after $120 \mathrm{~h}$ postinjection, demonstrating that TPAFN-F127 NPs can track tumors over a long period of time. Ex vivo fluorescence imaging revealed much stronger fluorescence in the liver and tumor, suggesting that TPAFN-F127 NPs possess tumor-targeting efficiency (Figure S11 and S12).The existence of TPAFNF127 NPs in the liver and spleen is due to the critical role of the reticuloendothelial system organs in the uptake and excretion of exogenous nanoparticles [47]. Furthermore, ex vivo fluorescence imaging of tissue slices was performed using fluorescence microscopy. As shown in Figure S13, no fluorescence was observed for the control group, but bright red spots were observed in the tissues of mice after intravenous injection with TPAFN-F127 NPs, which could be ascribed to the TPAFN-F127 NP signal.

2.4. Biodistribution of TPAFN-F127 NPs in Mice Revealed by LDI MSI. According to the intrinsic properties (multiple aromatic ring structures for good laser adsorption), TPAFN can be detected using LDI MS without any matrix, which greatly eliminates diffusion and improves the accuracy of position- ing. Figure S6 presents the typical LDI MS spectrum of TPAFN without any matrix assistance; the peaks at $\mathrm{m} / \mathrm{z}$ 564.2 and 487.1 correspond to the ions [TPAFN] $^{-}$and $\left[\text { TPAFN- } \mathrm{C}_{6} \mathrm{H}_{5}\right]^{-}$, respectively. Moreover, TPAFN-F127 NPs exhibited the same LDI MS signal as pure TPAFN, which indicated that Pluronic F127 matrix encapsulation did not change the intrinsic molecular weight of TPAFN, and TPAFN-F127 NPs could also be determined by LDI MS (Figure 5(a)). No ion signal was detectable in any tissue of blank mice by LDI MS without matrix assistance (Figure S14). By integrating the results from the control group with high physicochemical stability of TPAFN-F127 NPs, the expected $\mathrm{m} / \mathrm{z} 564.2$ and 487.1 in tissues (kidney, spleen, lung, liver, heart, and brain) of TPAFN-F127 NPinjected mice could be ascribed to the ion signal originating from injected TPAFN-F127 NPs (Figure S15). Further LDI MSI experiments were performed to quantify the deposition amount and analyze the biodistribution of TPAFN-F127 NPs in various organs. As shown in Figures 5(b) and 5(c), TPAFN-F127 NPs were clearly observed via active strong-toweak sequences of various organs. The variation of ion signal intensity in various tissue slices was consistent with ex vivo fluorescence imaging, indicating that the reciprocation was realized. More importantly, it is evident that the integration of LDI MSI and fluorescence imaging enhanced both the reliability and accuracy of determination. TPAFN-F127 NPs in various organs were quantified using the calibration curve acquired from each tissue spiked with TPAFN-F127 NPs (see details in Figure S16). The results showed that TPAFNF127 NPs mainly accumulated in the liver compared to other organs. In particular, no detectable fingerprint peaks of TPAFN-F127 NPs were found in the brain, which can be attributed to the low efficiency of TPAFN-F127 NPs across the blood-brain barrier. This result is consistent with those of fluorescence imaging.

2.5. Suborgan LDI MSI of TPAFN-F127 NPs. Owing to the limitations of strong background and poor penetration depth, it is very difficult for fluorescence technology to image the distribution of fluorescent nanoparticles in finer tissue structures. As a complementary technique, LDI MSI is 


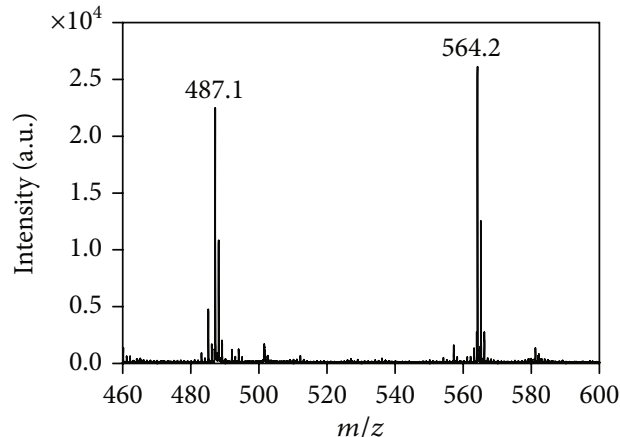

(a)

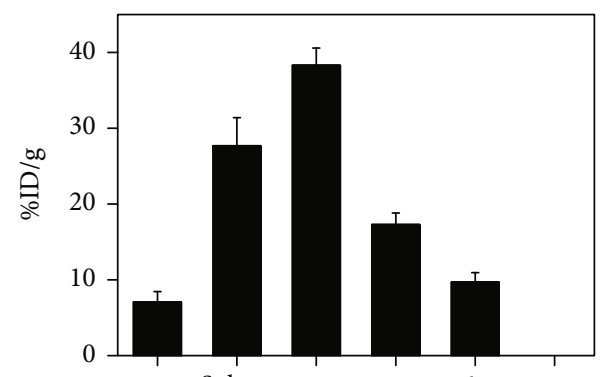

(b)

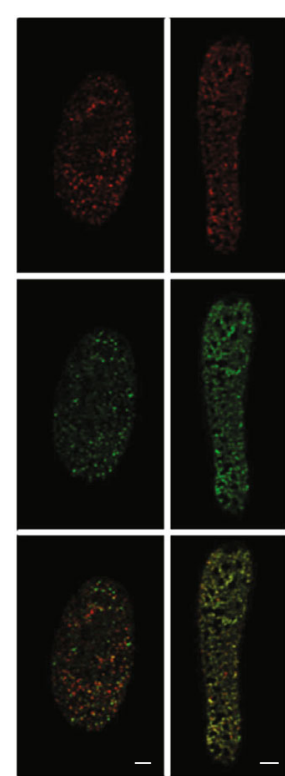

Kidney
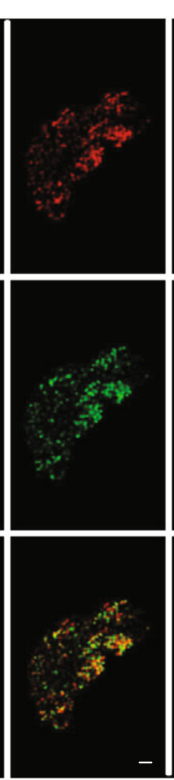

Spleen
Lung
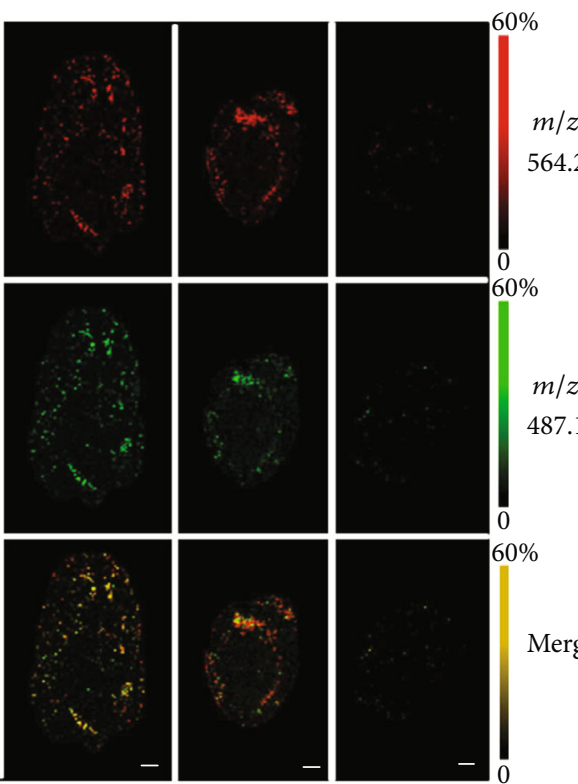

Liver

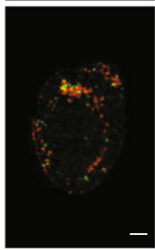

Heart

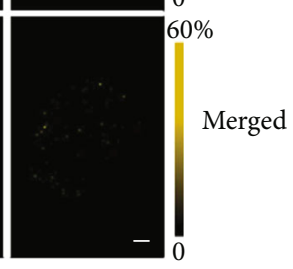

(c)

Brain

FIgURE 5: (a) Typical LDI MS spectrum of TPAFN-F127 NPs. (b) Quantification of TPAFN-F127 NPs in various mouse organs detected by LDI MS. \%ID/g denotes the percentage of injected dose per gram of tissue. (c) LDI MS images reveal the biodistribution of TPAFN-F127 NPs in different organs. Scale bars: $1 \mathrm{~mm}$.

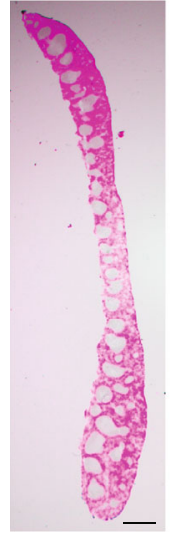

(a)

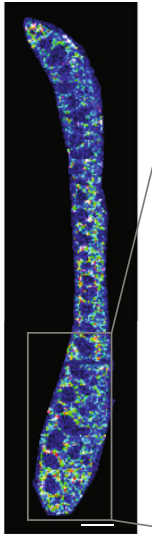

(b)

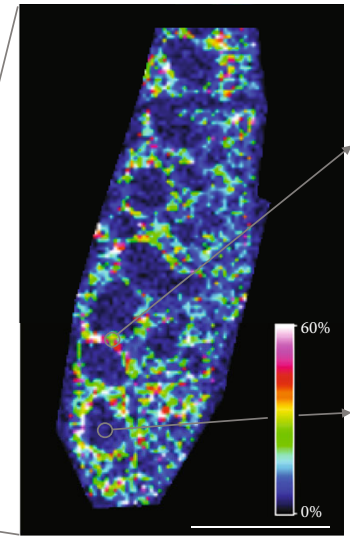

(c)
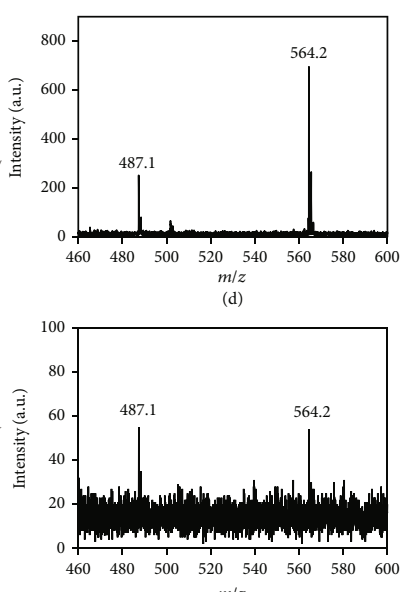

(e)

Figure 6: Suborgan distribution of TPAFN-F127 NPs in spleen tissue of a mouse. (a) Optical image of a spleen tissue slice. (b) Heat map to reveal the distribution $(\mathrm{m} / z$ 564.2) of TPAFN-F127 NPs in the spleen tissue slice. (c) Enlarged image of (b) indicates the distribution of TPAFN-F127 NPs in distinct histological regions. Scale bars: $5 \mathrm{~mm}$. (d) Representative LDI MS of red pulp region. (e) Representative LDI MS of white pulp region.

capable of revealing the chemical information and spatial distributions of target analytes within tissues. The suborgan distribution of TPAFN-F127 NPs in the spleen was characterized as a sample because the splenic tissue possesses distinguishable histological regions, including white pulp, red pulp, and a marginal zone (Figure S17) [48]. It is clear that the red pulp accumulated with a large number of TPAFN-F127 NPs, which could be because the spleen is responsible for blood filtration and clearance (Figure 6(b)) [48]. However, the deposition of TPAFN-F127 NPs in the white pulp region was much lower and even undetectable (Figure 6(e)). Furthermore, the marginal zone, the region between lymphoid white pulp and nonlymphoid red pulp, captures particulate antigens from circulation and presents antigens to lymphocytes of the spleen. Not surprisingly, a high ion intensity was found in these regions, and the quantitative 
results also support this conclusion (Figure 6(d) and Figure S18). This fine suborgan distribution of TPAFNF127 NPs was first studied by LDI MSI, which provides insight into the interaction between AIE NPs and living systems.

\section{Conclusion}

In summary, we prepared AIEgen-containing luminescent nanoparticles (TPAFN-F127 NPs) with superior physicochemical properties and excellent biocompatibility, making them preferable for biological imaging. Fluorescence imaging verified that TPAFN-F127 NPs are promising as a fluorescent probe in cell imaging and tumor targeting by the EPR effect. The distribution and quantification of TPAFN-F127 NPs were investigated by LDI MSI, which demonstrated that TPAFN-F127 NPs were mainly distributed in the liver, spleen, and lung. Detailed suborgan analysis of the spleen revealed that TPAFN-F127 NPs were heavily deposited more in the marginal zone and red pulp. Therefore, dual-modality imaging technology that combines fluorescence imaging with LDI MSI could be a good candidate for studying and assessing AIEgen-based nanoparticles before clinical biomedical applications. It is worth noting that this combination method has further potential implications, such as interaction analysis (e.g., modification and oxidation) between AIE nanoparticles and endogenous molecules, depending on the comprehensive molecular analysis capability by mass spectrometry.

\section{Materials and Methods}

4.1. Synthesis of 2,3-Bis[4-(diphenylamino)phenyl]fumaronitrile (TPAFN). Diphenylamine (338 mg, $2.0 \mathrm{mmol}), \mathrm{FN}-2 \mathrm{Br}(194$ $\mathrm{mg}, 0.5 \mathrm{mmol}), \mathrm{Pd}(\mathrm{OAc})_{2}(11 \mathrm{mg}, 0.05 \mathrm{mmol})$, and $\mathrm{Cs}_{2} \mathrm{CO}_{3}$ $(1.2 \mathrm{~g}, 3.5 \mathrm{mmol})$ were dissolved in $30 \mathrm{~mL}$ toluene, and then, tri-tert-butylphosphine $(30 \mathrm{mg}, 0.15 \mathrm{mmol}$ ) was added under an $\mathrm{N}_{2}$ atmosphere. The reaction mixture was stirred at $110^{\circ} \mathrm{C}$ and monitored by thin-layer chromatography (TLC). After reaction completion, the mixture was extracted with ethyl acetate. Finally, the crude product was purified by column chromatography (petroleum ether/ethyl acetate: 10/1) to yield TPAFN: ${ }^{1} \mathrm{H}$ NMR (400 MHz, $\mathrm{d}_{6}$-DMSO): $7.71-7.62$ $(m, J=7.68 \mathrm{~Hz}, 4 \mathrm{H}), 7.42-7.37(m, J=7.39 \mathrm{~Hz}, 8 \mathrm{H}), 7.21-$ $7.14(m, J=7.18 \mathrm{~Hz}, 12 \mathrm{H}), 6.97-6.94(m, J=6.95 \mathrm{~Hz}, 4 \mathrm{H})$; ${ }^{13} \mathrm{C}$ NMR (101 MHz, $\mathrm{d}_{6}$-DMSO): 150.55, 146.73, 146.43 , $129.92,129.74,126.02,124.80,124.61,121.02$, and 120.42; HRMS (MALDI-TOF, $m / z$ ): calculated for $\mathrm{C}_{40} \mathrm{H}_{28} \mathrm{~N}_{4}=$ 564.231 ; found $=564.150[\mathrm{M}]^{-}$.

4.2. Preparation of TPAFN-F127 NPs. The TPAFN-F127 NPs were prepared using the thin-film hydration method. In brief, Pluronic F127 $(20 \mathrm{mg})$ and TPAFN $(2 \mathrm{mg})$ were dissolved in $2 \mathrm{~mL}$ of THF under continuous sonication for 30 min. THF was then evaporated on a rotary evaporator at $40^{\circ} \mathrm{C}$. Finally, $2 \mathrm{~mL}$ of deionized water was added and the mixture was sonicated for $5 \mathrm{~min}$. After purification by a 0.2 $\mu \mathrm{m}$ filter, the obtained TPAFN-F127 NPs were stored at $4^{\circ} \mathrm{C}$. The concentration of TPAFN-F127 NPs was calculated using a UV/VIS/NIR spectrophotometer at $493 \mathrm{~nm}$.

\section{Data Availability}

The authors declare that all relevant data are included in this article and its supplementary information file. The remaining data are available from the corresponding authors upon request.

\section{Conflicts of Interest}

The authors declare no competing financial interests.

\section{Authors' Contributions}

X. Zhang, Z. Nie, and Y. Wei conceived and designed the experiments. L. Mao and Y. Jiang performed the experiments. H. Ouyang and Y. Feng helped with blood biochemical analysis. L. Mao, Y. Jiang, and R. Li analyzed the data and wrote the paper. All authors discussed the results and commented on the manuscript. Liucheng Mao and Yuming Jiang contributed equally to this work.

\section{Acknowledgments}

This research was supported by the National Natural Science Foundation of China (No. 21788102).

\section{Supplementary Materials}

Scheme S1: the synthetic route of AIE molecule (TPAFN). Scheme S2: the preparation of TPAFN-F127 NPs via a thinfilm hydration method. Figure S1: $1 \mathrm{H}$ NMR spectrum of FN-2Br. Figure S2: 13C NMR spectrum of FN-2Br. Figure S3: MALDI-TOF MS spectrum of FN-2Br. Figure S4: $1 \mathrm{H}$ NMR spectrum of TPAFN. Figure S5: 13C NMR spectrum of TPAFN. Figure S6: MALDI-TOF MS spectrum of TPAFN. Figure S7: TEM image of TPAFN-F127 NPs. Figure S8: the variation of fluorescent intensity of TPAFN-F127 NPs incubated with different mediums with various $\mathrm{pH}$ buffers. Table S1: changes of hematology parameters of mice induced by TPAFN-F127 NPs $(n=8)$. Figure S9: CLSM imaging of L929 cells after incubation with TPAFN-F127 NPs for $3 \mathrm{~h}$ at $37^{\circ} \mathrm{C}$. The concentration of TPAFN-F127 NPs is $5 \mu \mathrm{g}$ $\mathrm{mL}^{-1}$. Scale bar: $20 \mu \mathrm{m}$. Figure S10: variation in the intensity at different time points after tail-vein injection of TPAFNF127 NPs. $I_{0}$ refers to the intensity of the tumor region in the control group. Figure S11: ex vivo fluorescence imaging of tumor and major organs after $24 \mathrm{~h}$ postintravenous injection of TPAFN-F127 NPs $(200 \mu \mathrm{L}, 10 \mathrm{mg} / \mathrm{kg})$. Figure S12: histogram of the PL intensity of the organs (heart, liver, spleen, lung, and kidney), brain, and tumor. Figure S13: representative ex vivo fluorescence imaging of various types of tissue slices from mice treated with TPAFN-F127 NPs (200 $\mu \mathrm{L}, 10 \mathrm{mg} / \mathrm{kg}$ ) after $24 \mathrm{~h}$ postinjection; scale bar: $200 \mu \mathrm{m}$. Figure S14: representative LDI mass spectra of normal mouse tissue slice of the liver, spleen, lung, kidney, heart, and brain in negative ion mode. Figure S15: representative LDI mass spectra of TPAFN-F127 NP-injected normal mouse tissue 
slice of the liver, spleen, lung, kidney, heart, and brain in negative ion mode. Figure S16: standard calibration curves for TPAFN-F127 NPs in various organs including the liver, heart, lung, kidney, and spleen. Figure S17: photograph of splenic tissues. Figure S18: quantitative results of TPAFNF127 NPs in the white pulp, red pulp, and marginal zones in the spleen tissue section. (Supplementary Materials)

\section{References}

[1] M.-K. Tsang, G. Bai, and J. Hao, "Stimuli responsive upconversion luminescence nanomaterials and films for various applications," Chemical Society Reviews, vol. 44, no. 6, pp. 1585-1607, 2015.

[2] Y. Hong, J. W. Y. Lam, and B. Z. Tang, “Aggregation-induced emission," Chemical Society Reviews, vol. 40, no. 11, pp. 53615388, 2011.

[3] Z. Guo, C. Yan, and W.-H. Zhu, "High-performance quinoline-malononitrile core as a building block for the diversity-oriented synthesis of AIEgens," Angewandte Chemie International Edition, vol. 59, no. 25, pp. 9812-9825, 2020.

[4] F. Arcudi, L. Đorđević, and M. Prato, "Rationally designed carbon nanodots towards pure white-light emission," Angewandte Chemie International Edition, vol. 129, no. 15, pp. 4234-4237, 2017.

[5] Z. Zhao, J. W. Y. Lam, and B. Z. Tang, "Tetraphenylethene: a versatile AIE building block for the construction of efficient luminescent materials for organic light-emitting diodes," Journal of Materials Chemistry, vol. 22, no. 45, pp. 23726-23740, 2012.

[6] R. D. Costa, E. Ortí, H. J. Bolink, F. Monti, G. Accorsi, and N. Armaroli, "Luminescent ionic transition-metal complexes for light-emitting electrochemical cells," Angewandte Chemie International Edition, vol. 51, no. 33, pp. 8178-8211, 2012.

[7] H. S. Jung, P. Verwilst, A. Sharma, J. Shin, J. L. Sessler, and J. S. Kim, "Organic molecule-based photothermal agents: an expanding photothermal therapy universe," Chemical Society Reviews, vol. 47, no. 7, pp. 2280-2297, 2018.

[8] Z. Zheng, T. Zhang, H. Liu et al., "Bright near-infrared aggregation-induced emission luminogens with strong twophoton absorption, excellent organelle specificity, and efficient photodynamic therapy potential," ACS Nano, vol. 12 , no. 8 , pp. 8145-8159, 2018.

[9] S. N. Baker and G. A. Baker, "Luminescent carbon nanodots: emergent nanolights," Angewandte Chemie International Edition, vol. 49, no. 38, pp. 6726-6744, 2010.

[10] Y. Fan, P. Wang, Y. Lu et al., "Lifetime-engineered NIR-II nanoparticles unlock multiplexed in vivo imaging," Nature Nanotechnology, vol. 13, no. 10, pp. 941-946, 2018.

[11] J. Mei, N. L. C. Leung, R. T. K. Kwok, J. W. Y. Lam, and B. Z. Tang, "Aggregation-induced emission: together we shine, united we soar!," Chemical Reviews, vol. 115, no. 21, pp. 11718-11940, 2015.

[12] K. Li and B. Liu, "Polymer-encapsulated organic nanoparticles for fluorescence and photoacoustic imaging," Chemical Society Reviews, vol. 43, no. 18, pp. 6570-6597, 2014.

[13] D. Ding, K. Li, B. Liu, and B. Z. Tang, "Bioprobes based on AIE fluorogens," Accounts of Chemical Research, vol. 46, no. 11, pp. 2441-2453, 2013.
[14] Z. Sheng, B. Guo, D. Hu et al., "Bright aggregation-inducedemission dots for targeted synergetic NIR-II fluorescence and NIR-I photoacoustic imaging of orthotopic brain tumors," Advanced Materials, vol. 30, no. 29, p. 1800766 , 2018.

[15] S. Liu, C. Chen, Y. Li et al., "Constitutional isomerization enables bright NIR-II AIEgen for brain-inflammation imaging," Advanced Functional Materials, vol. 30, no. 7, p. $1908125,2020$.

[16] J. Qi, C. Sun, D. Li et al., “Aggregation-induced emission luminogen with near-infrared-II excitation and near-infrared-I emission for ultradeep intravital two-photon microscopy," ACS Nano, vol. 12, no. 8, pp. 7936-7945, 2018.

[17] X. Ni, X. Zhang, X. Duan, H. L. Zheng, X. S. Xue, and D. Ding, "Near-infrared afterglow luminescent aggregation-induced emission dots with ultrahigh tumor-to-liver signal ratio for promoted image-guided cancer surgery," Nano Letters, vol. 19, no. 1, pp. 318-330, 2019.

[18] C. Chen, X. Ni, H.-W. Tian, Q. Liu, D. S. Guo, and D. Ding, "Calixarene-based supramolecular AIE dots with highly inhibited nonradiative decay and intersystem crossing for ultrasensitive fluorescence image-guided cancer surgery," Angewandte Chemie International Edition, vol. 59, no. 25, pp. 10008 10012, 2020.

[19] J. Li, H. Gao, R. Liu et al., "Endoplasmic reticulum targeted AIE bioprobe as a highly efficient inducer of immunogenic cell death," Science China-Chemistry, vol. 63, no. 10, pp. 14281434, 2020.

[20] G. Feng and B. Liu, "Aggregation-induced emission (AIE) dots: emerging theranostic nanolights," Accounts of Chemical Research, vol. 51, no. 6, pp. 1404-1414, 2018.

[21] X. Cai, D. Mao, C. Wang, D. Kong, X. Cheng, and B. Liu, "Multifunctional liposome: a bright AIEgen-lipid conjugate with strong photosensitization," Angewandte Chemie International Edition, vol. 57, no. 50, pp. 16396-16400, 2018.

[22] D. Wang, M. M. S. Lee, W. Xu et al., "Boosting nonradiative decay to do useful work: development of a multimodality theranostic system from an AIEgen," Angewandte Chemie International Edition, vol. 58, no. 17, pp. 56285632, 2019.

[23] X. Gu, X. Zhang, H. Ma et al., "Corannulene-incorporated AIE nanodots with highly suppressed nonradiative decay for boosted cancer phototheranostics in vivo," Advanced Materials, vol. 30, no. 26, p. 1801065, 2018.

[24] F. Hu, S. Xu, and B. Liu, "Photosensitizers with aggregationinduced emission: materials and biomedical applications," Advanced Materials, vol. 30, no. 45, p. 1801350, 2018.

[25] N. Alifu, A. Zebibula, J. Qi et al., "Single-molecular nearinfrared-II theranostic systems: ultrastable aggregationinduced emission nanoparticles for long-term tracing and efficient photothermal therapy," ACS Nano, vol. 12, no. 11, pp. 11282-11293, 2018.

[26] C. Chen, H. Ou, R. Liu, and D. Ding, "Regulating the photophysical property of organic/polymer optical agents for promoted cancer phototheranostics," Advanced Materials, vol. 32, no. 3, p. 1806331, 2020.

[27] G. Feng, G.-Q. Zhang, and D. Ding, "Design of superior phototheranostic agents guided by Jablonski diagrams," Chemical Society Reviews, vol. 49, no. 22, pp. 8179-8234, 2020.

[28] C. Chen, X. Ni, S. Jia et al., "Massively evoking immunogenic cell death by focused mitochondrial oxidative stress using an 
AIE luminogen with a twisted molecular structure," Advanced Materials, vol. 31, no. 52, p. 1904914, 2019.

[29] B. He, B. Situ, Z. Zhao, and L. Zheng, "Promising applications of AIEgens in animal models," Small Methods, vol. 4, no. 4, p. 1900583, 2020.

[30] W. Yu, B. Guo, H. Zhang et al., "NIR-II fluorescence in vivo confocal microscopy with aggregation-induced emission dots," Science Bulletin, vol. 64, no. 6, pp. 410-416, 2019.

[31] W. Xu, D. Wang, and B. Z. Tang, "NIR-II AIEgens: a win-win integration towards bioapplications," Angewandte Chemie International Edition, vol. 59, pp. 2-14, 2020.

[32] K. A. Zemski Berry, J. A. Hankin, R. M. Barkley, J. M. Spraggins, R. M. Caprioli, and R. C. Murphy, "MALDI imaging of lipid biochemistry in tissues by mass spectrometry," Chemical Reviews, vol. 111, no. 10, pp. 6491-6512, 2011.

[33] K. Chughtai and R. M. A. Heeren, "Mass spectrometric imaging for biomedical tissue analysis," Chemical Reviews, vol. 110, no. 5, pp. 3237-3277, 2010.

[34] D. S. Cornett, M. L. Reyzer, P. Chaurand, and R. M. Caprioli, "MALDI imaging mass spectrometry: molecular snapshots of biochemical systems," Nature Methods, vol. 4, no. 10, pp. 828-833, 2007.

[35] K. Schwamborn and R. M. Caprioli, "Molecular imaging by mass spectrometry-looking beyond classical histology," Nature Reviews Cancer, vol. 10, no. 9, pp. 639-646, 2010.

[36] B. Prideaux and M. Stoeckli, "Mass spectrometry imaging for drug distribution studies," Journal of Proteomics, vol. 75, no. 16, pp. 4999-5013, 2012.

[37] M. Kompauer, S. Heiles, and B. Spengler, "Atmospheric pressure MALDI mass spectrometry imaging of tissues and cells at 1.4- $\mu \mathrm{m}$ lateral resolution," Nature Methods, vol. 14, no. 1, pp. 90-96, 2017.

[38] S. Chen, C. Xiong, H. Liu et al., "Mass spectrometry imaging reveals the sub-organ distribution of carbon nanomaterials," Nature Nanotechnology, vol. 10, no. 2, pp. 176-182, 2015.

[39] J. Xue, H. Liu, S. Chen et al., "Mass spectrometry imaging of the in situ drug release from nanocarriers," Science Advances, vol. 4, no. 10, p. eaat9039, 2018.

[40] B. Yan, S. T. Kim, C. S. Kim et al., "Multiplexed imaging of nanoparticles in tissues using laser desorption/ionization mass spectrometry," Journal of the American Chemical Society, vol. 135, no. 34, pp. 12564-12567, 2013.

[41] G. Feng, J. L. Y. Li, C. Claser et al., "Dual modal ultra-bright nanodots with aggregation-induced emission and gadoliniumchelation for vascular integrity and leakage detection," Biomaterials, vol. 152, pp. 77-85, 2018.

[42] K. Li, D. Ding, C. Prashant et al., "Gadolinium-functionalized aggregation-induced emission dots as dual-modality probes for cancer metastasis study," Advanced Healthcare Materials, vol. 2, no. 12, pp. 1600-1605, 2013.

[43] K. Li, W. Qin, D. Ding et al., "Photostable fluorescent organic dots with aggregation-induced emission (AIE dots) for noninvasive long-term cell tracing," Scientific Reports, vol. 3, no. 1, p. 1150, 2013.

[44] W. Qin, D. Ding, J. Liu et al., "Biocompatible nanoparticles with aggregation-induced emission characteristics as far-red/near-infrared fluorescent bioprobes for in vitro and in vivo imaging applications," Advanced Functional Materials, vol. 22, no. 4, pp. 771-779, 2012.
[45] H.-S. Peng and D. T. Chiu, "Soft fluorescent nanomaterials for biological and biomedical imaging," Chemical Society Reviews, vol. 44, no. 14, pp. 4699-4722, 2015.

[46] Y. Cai, W. Si, W. Huang, P. Chen, J. Shao, and X. Dong, "Organic dye based nanoparticles for cancer phototheranostics," Small, vol. 14, no. 25, p. 1704247, 2018.

[47] K. M. Tsoi, S. A. MacParland, X.-Z. Ma et al., "Mechanism of hard-nanomaterial clearance by the liver," Nature Materials, vol. 15, no. 11, pp. 1212-1221, 2016.

[48] R. E. Mebius and G. Kraal, "Structure and function of the spleen," Nature Reviews Immunology, vol. 5, no. 8, pp. 606616, 2005. 